\title{
Nature of the Supercritical Mesophase
}

\author{
Hamza J. Magnier1, Robin A. Curtis' ${ }^{1}$, Leslie V. Woodcock ${ }^{2}$ \\ ${ }^{1}$ Manchester Institute of Biotechnology, University of Manchester, Manchester, UK \\ ${ }^{2}$ Department of Physics, University of Algarve, Faro, Portugal \\ Email: hamza.javarmagnier@postgrad.manchester.ac.uk, r.curtis@manchester.ac.uk, lvwoodcock@ualg.pt
}

Received 2 May 2014; revised 26 May 2014; accepted 14 June 2014

Copyright (C) 2014 by authors and Scientific Research Publishing Inc.

This work is licensed under the Creative Commons Attribution International License (CC BY).

http://creativecommons.org/licenses/by/4.0/

c) (i) Open Access

\begin{abstract}
It has been reported that at temperatures above the critical there is no "continuity of liquid and gas", as originally hypothesized by van der Waals [1]. Rather, both gas and liquid phases, with characteristic properties as such, extend to supercritical temperatures [2]-[4]. Each phase is bounded by the locus of a percolation transition, i.e. a higher-order thermodynamic phase change associated with percolation of gas clusters in a large void, or liquid interstitial vacancies in a large cluster. Between these two-phase bounds, it is reported there exists a mesophase that resembles an otherwise homogeneous dispersion of gas micro-bubbles in liquid (foam) and a dispersion of liquid micro-droplets in gas (mist). Such a colloidal-like state of a pure one-component fluid represents a hitherto unchartered equilibrium state of matter besides pure solid, liquid or gas. Here we provide compelling evidence, from molecular dynamics (MD) simulations, for the existence of this supercritical mesophase and its colloidal nature. We report preliminary results of computer simulations for a model fluid using a simplistic representation of atoms or molecules, i.e. a hard-core repulsion with an attraction so short that the atoms are referred to as "adhesive spheres". Molecular clusters, and hence percolation transitions, are unambiguously defined. Graphics of color-coded clusters show colloidal characteristics of the supercritical mesophase. We append this Letter to Natural Science with a debate on the scientific merits of its content courtesy of correspondence with Nature (Appendix).
\end{abstract}

\section{Keywords}

Supercritical Mesophase, Adhesive-Sphere, Criticality, Liquid-Gas

\section{Introduction}

Along supercritical isotherms, the densities at which molecular clusters of occupied sites in the gas phase, or clusters of unoccupied voids in the liquid phase, first become macroscopic, are referred to percolation transitions. The respective limiting percolation transitions, denoted by PB and PA, refer to "bonded cluster" and 
“available volume” for gas and liquid phases respectively [2]-[4]. For real fluids, however, with continuous Hamiltonians, there is no unique molecular distance parameter to define a cluster, hence PA and PB are not generally defined at the molecular level. The percolation loci can, nonetheless, be defined thermodynamically along supercritical isotherms by the conditions

$$
\begin{gathered}
\text { gasphase } \rho<\rho_{P B} \quad \text { then }\left(\mathrm{d}^{2} p / \mathrm{d} \rho^{2}\right)_{T}<0 \\
\text { mesophase } \rho_{P B}>\rho>\rho_{P A} \text { then }\left(\mathrm{d}^{2} p / \mathrm{d} \rho^{2}\right)_{T}=0 \\
\text { liquid phase } \rho>\rho_{P A} \text { then }\left(\mathrm{d}^{2} p / \mathrm{d} \rho^{2}\right)_{T}>0
\end{gathered}
$$

Thus, not only is there no continuity of liquid and gas, but the two equilibrium fluid states of matter are fundamentally different in their respective thermodynamic descriptions. Rigidity $\left[(\mathrm{d} p / \mathrm{d} \rho)_{T}\right]$ is inversely proportional to fluctuations in the atomic number density. For a gas of attractive atoms, rigidity decreases with density because fluctuations increase with polymerisation, whereas for a liquid, rigidity increases with density because the voids become fewer as density increases. This condition also holds true for the subcritical isotherms of liquid and gas when $T<T_{c}$. To observe this intermediate mesophase that is neither pure liquid nor gas, we have investigated a model fluid of cohesive atomic spheres that gives rise to well-defined molecular clusters.

The energy of interaction between two square-well molecules $i$ and $j$ separated by distance $r_{i j}$ is (see Figure 1 )

$$
\begin{gathered}
\varphi_{i j}(r)=0 \text { for } r_{i j}>\lambda \sigma \\
\varphi_{i j}(r)=-\varepsilon \text { for } \sigma>r_{i j}>\lambda \sigma \\
\varphi_{i j}(r)=\infty \text { for } r_{i j}<\sigma
\end{gathered}
$$

where $\sigma$ is the hard-sphere diameter, $\varepsilon$ is the square-well depth, and $\lambda \sigma$ is the range. The reduced thermodynamic states are then temperature $T^{*}=k_{B} T / \varepsilon$ and density

$$
\rho^{*}=N \sigma^{3} / V
$$

where $k_{B}$ is Boltzmann's constant and $N / V$ is the number density.

Interestingly, there is also an experimental manifestation of this model system in solutions of colloidal or protein molecules whose osmotic properties share a corresponding states law within McMillan-Mayer theory, with essentially the same phase diagram as molecular fluids [5] [6]. The adhesive model presented here is more relevant for charged colloids or proteins dissolved in aqueous solutions at moderate ionic strength. Under these conditions, the range of the pair potential is much smaller than the colloid diameter.

When $\varepsilon \rightarrow 0$ the hard-sphere fluid is recovered. The percolation transitions of the hard-sphere fluid, as defined by Kratky [7], have been computed [8] and found to be weak higher-order thermodynamic phase, transitions that occurs as the fluctuations in either number density, or equivalently available volume, change at the percolation

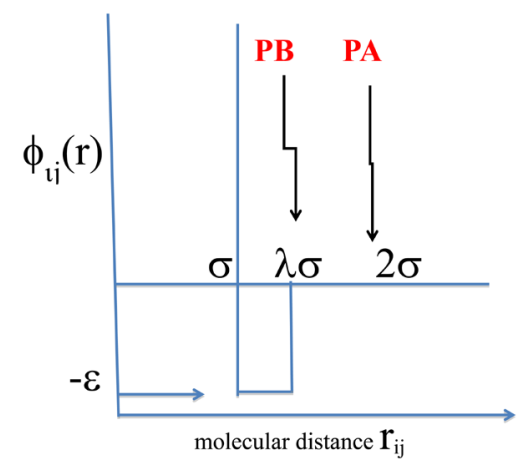

Figure 1. The square-well model atom-atom pair potential $\left[\varphi_{i j}\left(r_{i j}\right)\right]$ with characteristic distances that define clusters, and hence percolation transitions, in the fluid phases: the available volume percolation transition PA associated with the distance $2 \sigma$ is defined previously ([7] and [8]); PB is the percolation transition associated with bonded clusters of atoms within the distance $\lambda \sigma$. 
threshold. The transition in the hard-sphere fluid $(\varepsilon=0)$ is so weak that, until recently it has been imperceptible from the equations-of-state. The effect of any added attraction of finite $\varepsilon$, however, is to strengthen the percolation transition with discontinuities in fluctuation-related second-order properties, heat capacity and compressibility, with decreasing temperature. Eventually, with sufficient cooling, at a "critical temperature", the percolation loci intersect in the Gibbs pressure-temperature $(p-T)$ plane. At this point, a liquid state at the density of the available-volume percolation transition coexists in thermodynamic equilibrium with a vapor state at lower density of a percolation transition associated with bonded clusters of gas molecules.

\section{Molecular Dynamics}

We have performed extensive molecular dynamics (MD) simulations, using a computer program DYNAMO, along several supercritical isotherms of an adhesive sphere fluid for $\lambda=1.005$. A full report with details of these computations will be published elsewhere. DYNAMO is a modern event-driven MD simulations package developed by Bannerman [9] [10] and distributed under the General Public License. The full source code and documentation are freely available online at DYNAMO's own web domains including dynamod.org, dynamod.co.uk and dynamod.com. DYNAMO sets out to calculate the smallest time to the next event. Within this time, the velocity trajectory of the events is then calculated by solving Newton's equation of motion for the next event, e.g. collision of capture between any two atoms.

The results of the simulations are shown for the square-well fluid system of $N=10976$ particles, i.e. $(14 \times 14$ $\times 14 \times 4$ ) fixed initially on an FCC lattice, with periodic boundary conditions. The pressures along a supercritical isotherm $T^{*}=0.215$, shown in Figure 2, are obtained from independent runs of 70 state points along the isotherm in the region shown. Every state point is equilibrated independently for 10 million collisions and then simulated for 1 billion collisions with ensemble averaging of thermodynamic properties with averages taken over $10^{13}$ collisions where pressure is collected. In all, we have studied a total of 700 state point for 8 isotherms going from $T=0.210 T / T_{c}=1.075$ until $T / T_{c}=5$. By interpolating the slope in the mesophase, $T_{c}$ can be determined and is found to be $0.200 \pm 0.002$.

There is a connection between two coexisting phases at the critical temperature, and the percolation transition loci. The coexisting vapor density follows the loci that one expects, not for the hard-sphere excluded-volume percolation, but the extended-volume bonded cluster percolation transition of the square well. At the critical temperature and pressure, the vapor is at the bonded cluster percolation density $\rho_{\mathrm{PB}}$, and the liquid at $\rho_{\mathrm{PA}}$. At this critical temperature, both gas and liquid have the same chemical potential as the gradient in chemical potential $(\mathrm{dp} / \rho)$ approaches zero for densities between $\rho_{\mathrm{PB}}$ and $\rho_{\mathrm{PA}}$ i.e. as the density fluctuations diverge. At very narrow well-width approaching the adhesive-sphere limit $(\lambda=1.005), \rho_{\mathrm{PE}}$ and $\rho_{\mathrm{PA}}$ are 0.30 and 0.45 respectively (Figure 2). This critical divide is narrower that for all other square-well fluids with wider attractive wells [2].

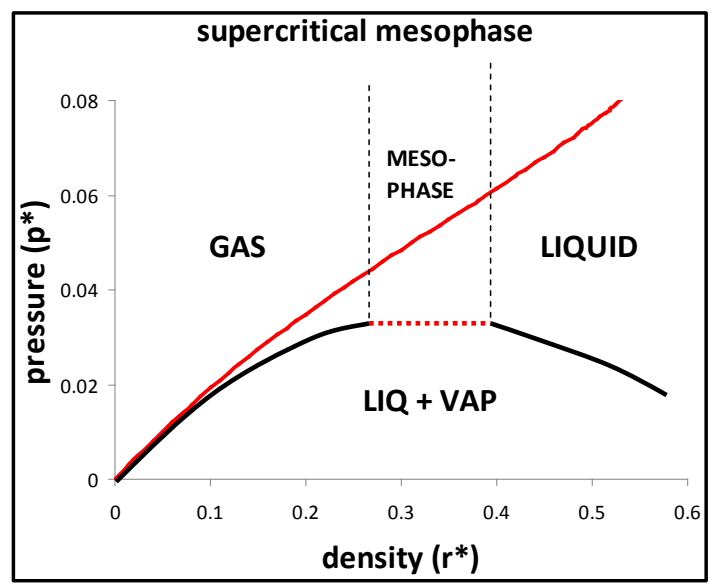

Figure 2. Supercritical isotherm of the adhesive square-well model fluid $(\lambda=1.005) T / T_{c}=1.075$; the critical divide is shown as a dashed red line at the critical temperature $T_{c}{ }^{*}=0.200 \pm 0.002$; the straight line is added to clarify the mesophase linear region and the percolation transitions that bound gas and liquid phases. 
MD configurations along the slightly supercritical isotherm $\left(T^{*}=0.215\right)$ shown in Figure 2 have been carried out to determine the probability distribution of finding a particle in a cluster of size $\mathrm{n}$ at equilibrium. At very low density, i.e. for an ideal gas, there are no clusters and

$$
n_{c} / N=1
$$

where $n_{c}$ is the number of clusters. As the density increases, there are an ever-increasing number of pairs, then triplets etc., and increasingly large molecular clusters, until reaching a density when just one cluster of size $N$ fills the whole box. At this point the probability function $\left(n_{c}-1\right) / N$ goes to zero for large $N$. The cluster distribution for the intermediate density $\rho^{*}=0.36$, i.e. within the region of the mesophase, is shown in Figure 3.

The density-dependent \% probability function $P(n)$ can be defined by

$$
\% P(n)=100 \times n_{c}(n) n / N
$$

where $n_{c}(n)$ is the number of clusters of size $n$. Multiplication by 100 is simply to avoid very small numbers on the log plots; the total probability normalizes to $100 \%$. This is a normalized probability of a cluster of size $n$.

Figure 3 shows that there is clear mixture of gas phase and liquid phase in the supercritical mesophase. The gas component at the density 0.36 is characterized by a distribution of molecular sizes, monomers $(12 \%)$ dimers (6\%), trimers (4\%), etc. with ever-decreasing probability, with an extremely small probability extending up to several hundred. The liquid phase, by contrast, shows just one cluster that is of the order of the size of the system, but variations in size in probability result as gas and liquid fractions fluctuate. The data in Figure 2 are indicative of a linear combination rule for thermodynamic state functions, which explains the linear equation-ofstate for the pressure $\mathrm{p}(\rho)_{\mathrm{T}}$ in the supercritical mesophase, as noted previously [2]-[4], and seen here in Figure 2, for example.

Another more illuminating way to reveal the colloidal nature of the mesophase is by computer graphics, using color-coding for clusters of different sizes. In Figure 4, we show pictures of the molecular structures along a supercritical isotherm for densities corresponding to the gas phase (4a), the mesophase, on the gas side (4b) the mesophase on the liquid side (4c), and liquid phase (4d).

Figures 4(a) shows the gas molecules with a predominance of monomers (red) and all other sized molecules (brown); the largest molecules are around 30 atoms at this density, albeit with very low probability.

Figure 4(b) represents an equilibrium configuration within the mesophase at the density $\rho^{*}=0.36$. The colorcoding for the gas phase is the same as in Figure 4(a), except that the largest gas cluster, 267 atoms, is shown as yellow. There are two very large liquid clusters in this particular snapshot colored dark blue (4042 atoms) and

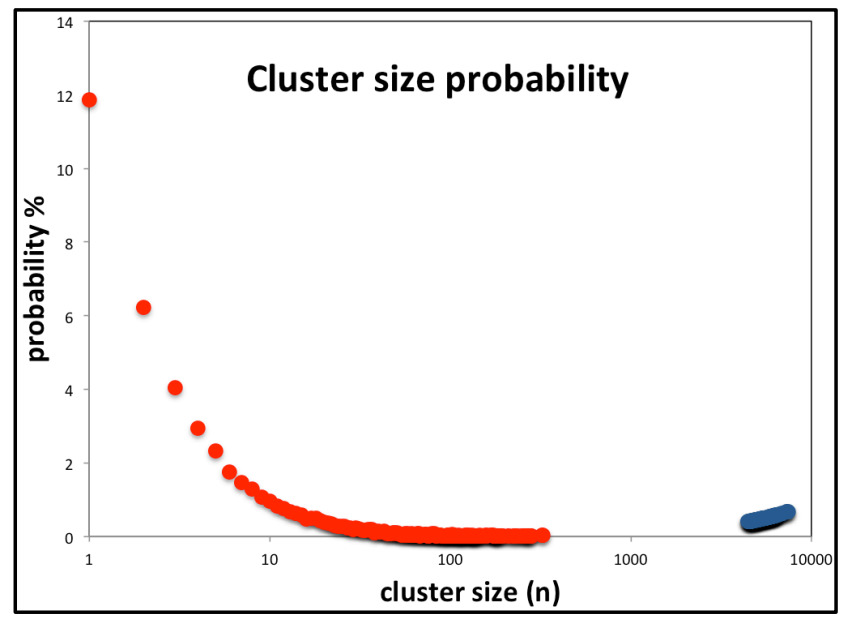

Figure 3. Distribution of clusters at the density $\rho^{*}=0.36$ for a supercritical isotherm at temperature $T^{*}=0.215$ (see Figure 2): the probability plot shows that all the atoms in the system belong either to a gas phase (molecules of less than 400) or liquid phase with clusters of the order of the system size $>5000$; the hiatus shows there is no intermediate cluster sizes, hence no "continuity between liquid and gas”. 


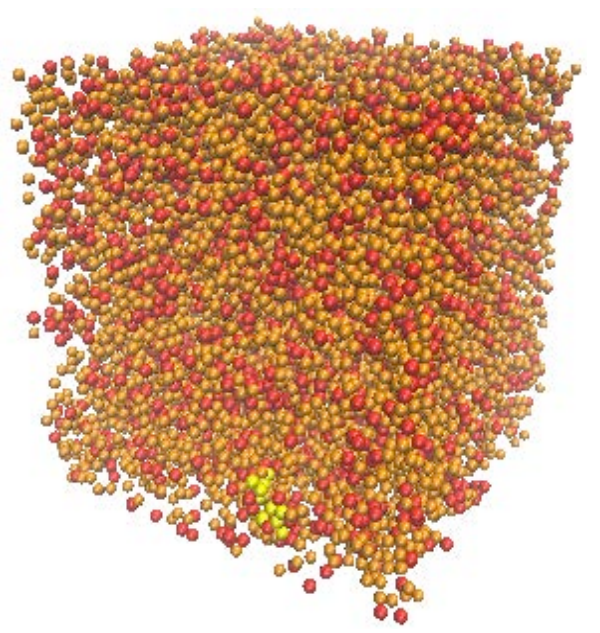

(a)

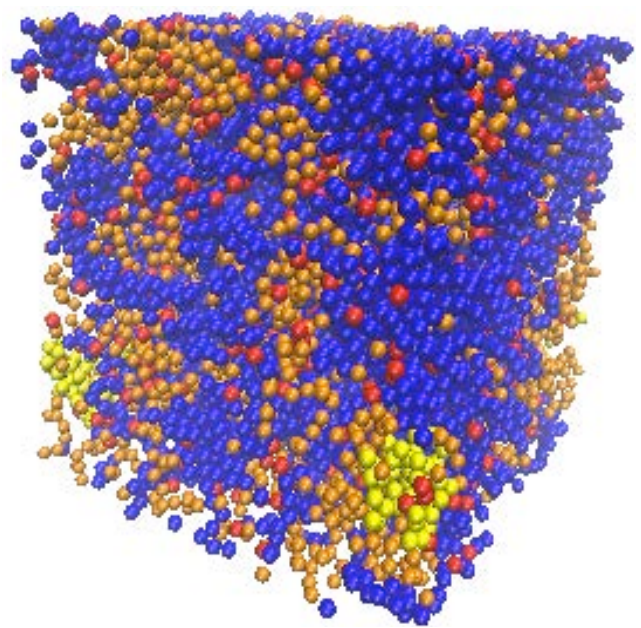

(c)

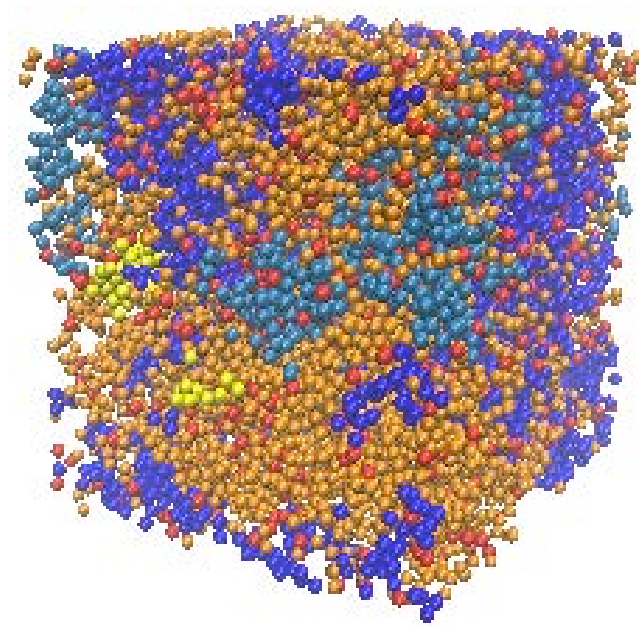

(b)

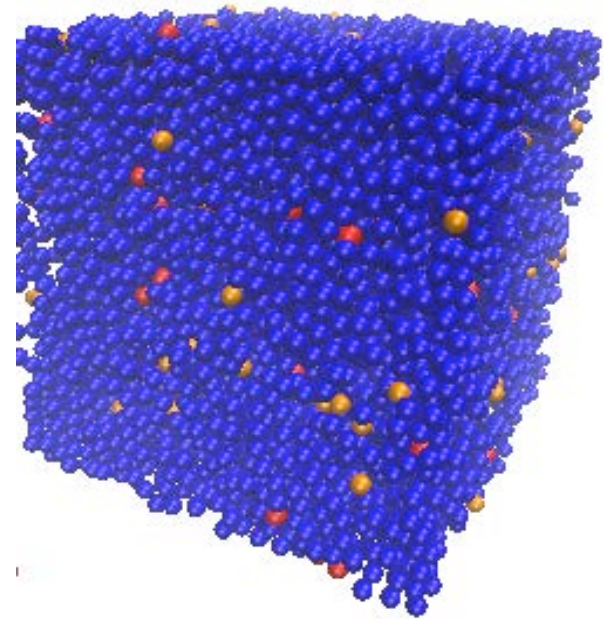

(d)

Figure 4. Snapshots of instantaneous configurations of the model fluid along a supercritical isotherm $T^{*}=0.215\left(T / T_{c}=1.075\right)$ Atoms as gas phase monomers are red, and molecules $(n \geq 2)$ brown; all atoms in liquid state clusters are blue; in the mesophase (4(b) and 4(c)), the largest clusters of gas molecules are green and yellow. (a) gas phase $\rho^{*}=0.2$; (b) mesophase gas-side $\rho^{*}=0.36$; (c) mesophase liquid-side $\rho^{*}=0.4$; (d) liquid phase $\rho^{*}=0.7$.

second cluster colored light blue (1129). The important point to note is that every atom or molecule can be designated liquid or gas phase. There are no clusters of an intermediate size, i.e. there is no "continuity of gaseous and liquid phases". We see a colloidal mixture that is nanoscopically heterogeneous but thermodynamically homogeneous, as distinct from the subcritical coexistence region that is thermodynamically heterogeneous.

In Figure 4(c), on the liquid side of the mesophase, $\rho^{*}=0.4$, the mixture of gas molecules and liquid cluster is also evident. The main difference between Figure 4(b) and Figure 4(c) is the gas side of the mesophase (4b), the dispersed phase is the liquid nanodroplets, (resembling a colloidal mist) whereas on the liquid side of the mesophase (4c) the dispersed phase is the nano-bubbles of gas (resembling a colloidal foam). Finally, for densities above the critical percolation threshold, as seen in Figure 4(d), there is just one large liquid cluster with a very dilute solution of gas like monomers or dimers.

\section{Conclusions}

In summary, recent discoveries of an alternative description of liquid-gas criticality have been extended for adhesive-sphere fluids. The revised phase diagram shows three equilibrium fluid phases. The "liquid" phase spans 
all temperatures from a metastable amorphous ground state to supercritical temperatures. There is a supercritical meso-phase bounded by two percolation transition loci and a gas phase. There is no "critical point" on the Gibbs density surface. When two percolation transitions have the same pressure, i.e. on intersection of the loci in the $p-T$ plane, an equilibrium dividing line between the supercritical mesophase and subcritical coexistence is thermodynamically defined.

We have obtained revised results of the critical temperature $\left(T_{c}\right)$ and critical pressure $\left(p_{c}\right)$ using only data from one-phase supercritical isotherms, and coexisting gas and liquid densities at $T_{c}$. Values of coexisting densities for adhesive sphere at $T_{c}$ and the density loci of the percolation transitions that bound the existence of supercritical gas and liquid phases for $T>T_{c}$. These results confirm that there exists a supercritical mesophase as a fourth equilibrium state of matter after crystal, liquid and gas, with characteristic properties of a single-component colloid phase of gas in liquid or liquid in gas.

Finally, we note that a colloidal nature of the supercritical mesophase is consistent with the well-known phenomenon of critical opalescence. The present interpretation of the thermodynamics of criticality provides an alternative description of critical opalescence [11] observed in both molecular liquids and protein solutions [12], known as Tyndall scattering, which is a manifestation of a colloidal nature arising from light scattering by colloidal particles with sizes comparable to the wavelengths of white light.

A detailed account of this research will be published elsewhere, and reported with more detail in the Ph.D. Thesis of HJM [13].

\section{Acknowledgements}

We wish to thank the University of Manchester for the award of a Research Scholarship to HJM, and to acknowledge many helpful discussions with Drs. Marcus Bannerman and Leo Lue.

\section{References}

[1] Van der Waals, J.D. (1873) Over de Continuiteit van den Gas-en Vloeistoftoestand. (On the Continuity of the Gas and Liquid State). Ph.D. Thesis, Leiden. (for English translation see Rowlinson, J.S., Dover Publications, 1982)

[2] Woodcock, L.V. (2012) Thermodynamic Description of Liquid-State Limits. The Journal of Physical Chemistry (B), 116, 3735-3744. http://dx.doi.org/10.1021/jp2116214

[3] Woodcock, L.V. (2013) Observations of a Thermodynamic Liquid-Gas Critical Coexistence Line and Supercritical Fluid Phase Bounds from Percolation Transition Loci. Fluid Phase Equilibria, 351, 25-33. http://dx.doi.org/10.1016/j.fluid.2012.08.029

[4] Heyes, D.M. and Woodcock, L.V. (2013) Critical and Supercritical Properties of Lennard-Jones Fluids. Fluid Phase Equilibria, 356, 301-308. http://dx.doi.org/10.1016/j.fluid.2013.07.056

[5] Curtis, R.A. and Lue, L. (2006) A Molecular Approach to Bioseparations, Protein-Protein and Protein-Salt Interactions. Chemical Engineering Science, 61, 907-923. http://dx.doi.org/10.1016/j.ces.2005.04.007

[6] McMillan, W.G. and Mayer, J.E. (1945) The Statistical Thermodynamics of Multicomponent Solutions. The Journal of Chemical Physics, 13, 276-305. http://dx.doi.org/10.1063/1.1724036

[7] Kratky, K. (1988) Is the Percolation Transition in the Hard-Sphere Fluid a Thermodynamic Phase Transition? Journal of Statistical Physics, 52, 1413-1421. http://dx.doi.org/10.1007/BF01011656

[8] Woodcock, L.V. (2011) Percolation Transitions in the Hard-Sphere Fluid. JAIChE, 58, 1610-1618.

[9] Bannerman, M.N. (2014) DynamO. http://dynamomd.org/

[10] Bannernman, M.N. and Lue, L. (2010) Exact On-Event Expressions for Discrete Potential Systems. The Journal of Chemical Physics, 133, Article ID: 124506. http://dx.doi.org/10.1063/1.3486567

[11] Gopal, E.S.R. (2000) Critical Opalescence. Resonance 37-45. (This prevailing explanation of critical opalescence as reviewed by Gopalrequires molecular fluctuations or Brownian motion, according to the theories of Einstein and Smoluchowski. It now seems likely that the effect may have little to do with molecular level fluctuations directly, and that there is a simpler explanation as Tyndall scattering which occurs due to the colloidal dispersion nature of the mesophase.) www.ias.ac.in/resonance/Apr2000/pdf/Apr2000p37-45.pdf http://www.chm.bris.ac.uk/webprojects2002/pdavies/Tyndall.html

[12] Mason, B.D., Zhang, L., Remmele, R.L.J. and Zhang, J. (2011) Opalescence of an IgG2 Monoclonal Antibody Solution as It Relates to Liquid-Liquid Phase Separation. Journal of Pharmaceutical Sciences, 100, 4587-4596. http://dx.doi.org/10.1002/jps.22650

[13] Magnier, H.J. (2014) Ph.D. Thesis, University of Manchester, Manchester. (In Preparation) 


\section{Appendix}

Correspondence with Nature (communicated April $1^{\text {st }}$ 2014): debate on the evidence in this Letter for the existence and nature of the supercritical mesophase.

Referee 1: "The main message is (as previously stated in the JCP (B) article) that the assumption of continuity of the thermodynamic functions and the theory of critical phenomena are wrong. This is an extremely strong message. If it were true, it would invalidate our understanding of phase transitions, including the exact results! For the two-d lattice-gas model indeed the exact Onsager analytic solution proves the absence of mesophases!"

Authors: The new science of criticality we confirm in this Letter does indeed invalidate the concept of "universality" between critical phenomena in Ising lattice gases and Gibbs thermodynamic liquid-gas systems. The reason is simply that Ising models are not "Gibbsian" thermodynamic systems capable of exchanging both heat and work as required by Gibb's definition for compliance with $1^{\text {st }}$ and $2^{\text {nd }}$ Laws, and upon which the Gibbs phase rule, for example, and Gibbs density $p-T$ surface phase bounds are derived. Ising models have no Gibbs chemical potential to define sharp first-order phase transitions characterized by latent heat and latent work (expansion or contraction). Onsager's exact solution for the 2-dimensional Ising lattice gas "proves" nothing about the nature of phase boundaries in real fluids.

Referee 1: Since the pressure is the derivative of the free energy, the authors are assuming that in fluids the free energy does not have continuous derivatives even far from the critical point (the only point where the free energy is truly non analytic according to critical phenomena!)

Authors: What free energy we ask? In a liquid-gas system at constant $T$ the pressure is indeed the derivative of Helmoltz energy with respect to a change in volume. For non-Gibbsian systems, such as an Ising lattice gas, however, (for which the referees statement about non-analytic phenomena in brackets is applicable) volume is neither a state function nor a state variable, there is no work, hence no $1^{\text {st }}$ or $2^{\text {nd }}$ Laws from which the definition of Gibbs chemical potential which Gibbs phase rule, and phase transition descriptions, are derived.

Referee 1: Now, I was expecting strong evidence in favor of the important claim made. But I did not find any evidence. The manuscript reports the equation of state at one single temperature and for one single model in which an apparent straight segment is shown. But there is no evidence of the discontinuities in the derivatives of the equation of state that would be the proof of the original statement.

Authors: This Nature letter is not about the thermodynamic order of the percolation transitions. It is about the colloidal "Nature of the Supercritical Mesophase." The referee is here "refereeing" the science in other papers (Reference [2]) that have already been refereed and published.

Referee 1: In this respect, I would conclude that all hypothesis (not to say speculations) were already presented in the JCP (B) and nothing is added by this Letter.

Authors: Unlike the van der Waals hypothesis, the description of criticality in JCP(B) contains no "hypothesis”, just a more plausible description of the phase bounds based upon our knowledge of percolation loci, simulations, and the experimental literature. Figure 4 in this Letter contains hitherto unpublished computer images of coexisting liquid-like and gas-like clusters in the supercritical mesophase. This is new compelling evidence for the existence and "nature of the supercritical mesophase". How can the referee justify the statement "nothing is added by this Letter?"

Referee 1: In addition, I like to mention some further points: the chosen potential (square well with a range 0.005 sigma) is not stable in the region where it has been studied. The stable phase is the crystal phase. The authors do not comment on this issue and do not provide any evidence that in their simulations partial crystallization has not taken place.

Authors: The overall phase diagram of this model has not yet been properly determined. If there were to be first-order crystallization it would be manifested as a sudden decrease in the pressure. There is no such evidence of instability in the fluid phases with respect to crystallization. The thermodynamic properties we calculate are entirely consistent with the three pure fluid phases that we see in the computer graphics in Figure 4 with pressure increasing monotonically with density.

Referee 1: Figure 3 provides misleading information. What is shown here is the cluster size distribution for one selected density. As we learn from percolation theory (the beautiful book of Stauffer, Introduction to Percolation Theory is a good reference) is that the cluster size distribution is a power-law with a cut off function whose characteristic size diverges at percolation. Beyond percolation, the characteristic size of the distribution goes back continuously to one. What the authors show is nothing more than the cluster size distribution beyond 
percolation (in this case I assume having defined a bond as a pair of particles with relative distance less than sigma + delta, even if it is not specified in the text, but only by the arrow in Figure 1) Indeed, entering inside the percolation region, the cluster size of the finite clusters trace back toward smaller and smaller clusters while a new peak appears for clusters of the order of the system size (the infinite cluster). If they had drawn curves for several rho (and in a log-log scale!!!) they would have clearly observed such continuity of behaviors and would have properly interpreted the result as the typical behavior of a percolating system. According to the authors we would conclude that every time there is percolation, then there is a mesophase!

Authors: Here is the plot on a log-log scale for the density $\rho^{*}=0.36$ within the mesophase; there is no "continuity”.

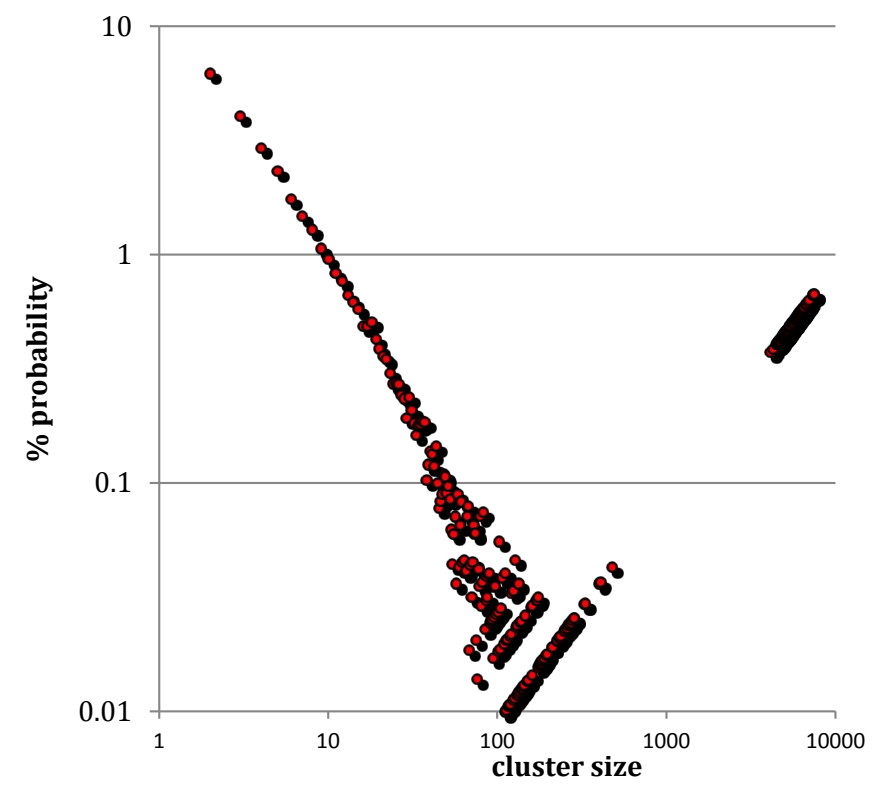

Cluster probability data from Figure 3 shown here on log-scale.

We have similar curves showing the hiatus for all densities in the mesophase; density changes within the mesophase make no difference to this aspect.

Referee 1: The final qualitative comments about the interpretation of the critical scattering in term of a colloidal phase completely neglects all we have learned in the last century about critical phenomena and the Coniglio-Klein mapping between percolation and criticality.

Authors: “Coniglio-Klein” mapping is a mathematical cluster contraption for describing percolation phenomena in constrained lattice systems, e.g. Potts or Ising models. The concept is not applied to the statistical description of percolation in liquid-gas thermodynamic equilibria, as originally defined by Gibbs.

Referee 1: I find a waste of the precious space of a Nature Letter that 10 lines of the manuscript (Page 3) are deserved to advertisement of a standard common event-driven MD code, as if the code were a breakthrough in computational physics. This type of code is very common and I can provide a very large number of references to event-driven simulations, even with potential more complex that a simple square well (SW)! A similarly comment applies to the cluster partitioning code, again a standard computational tool.

Authors: In describing the source of our computational results, we are merely complying with good science publishing protocol. Whether its real experiments or computer "experiments", essential technical details should be included so that any subsequent investigator can repeat our computations by downloading DYNAMO and checking our results, and indeed also reach the same conclusions.

Referee 1: Finally, I like to stress that there is no doubt that the structure of any supercritical fluid changes from gas-like to liquid-like (with a very large change of density involved).

Authors: At constant $T$, along any supercritical isotherm, the thermodynamic data show that change in density with pressure $(\mathrm{d} \rho / \mathrm{d} p)_{T}$ is constant within the broad mesophase region as the fractional ratio of liquid to gas increases from zero to 1 , whatever the thermodynamic description of criticality. 
Referee 1: And there is no doubt that this change is fastest at the Widom line, the line of compressibility maxima that emanates from the critical point (the equivalent of the $H=0$ line in the Ising analog of the lattice gas problem). And there is no doubt that close to the Widom line the equation of state looks linear.

Authors: There seems to be a variety of definitions in the literature of hitherto mysterious supercritical lines defined by various maxima in second-order thermodynamic properties and some dynamical and transport properties. The present description of supercriticality explains many of these observations.The reason for an apparent line of maximum isothermal compressibility $\rho^{-1}(\mathrm{~d} \rho / \mathrm{d} p)_{T}$, stemming from $p_{c}, T_{c}$ in the $p$-T plane is simply that the rigidity function $(\mathrm{d} p / \mathrm{d} \rho)_{T}$ decreases with density for a supercritical gas, and increases with density for the supercritical liquid at the same temperature, and hence has a constant minimum value within the mesophase. The $H=0$ line in the Ising model may be an interesting analogy, butthis is not a "thermodynamic" system in Gibbs-Duhem definition and hence will behave with different characteristics at a critical singularity.

Referee 1: In summary, if the authors wanted to convince the community of the existence of a new phase they should have shown data based on their SW model simulation, that unambiguously prove the existence of discontinuities in the third derivative of $p(\rho)$. Everything else is, in my opinion, only a speculation.

Authors: Thermodynamics is an empirical science; it is not possible to "prove" anything regarding the behavior of state functions on a Gibbs surface. One can only provide compelling evidence for a more plausible description and scientific interpretation of phase bounds. More plausible, for example, than van der Waals hypothetical "critical point" on the Gibbs density surface, that lacks any thermodynamic definition, and is inconsistent with Gibbs phase rule as derived from the laws of thermodynamics, and indeed also with experimental thermodynamic measurements at $T_{c}$.

The existence of a supercritical mesophase is already established science as evidenced by the various published papers during the last two years e.g. reference [2] of the Letter. This Letter to Nature however, is not about the order of the thermodynamic phase transitions associated with percolation loci, it is about the pictorial evidence for the colloidal nature of the mesophase. Figure 4 shows quite unambiguously, that there exists a mesophase twixt gas and liquid on supercritical isotherms, that is neither gas nor liquid, but with characteristics of a colloidal dispersion of one phase in the other.

Referee 2: The authors report the results of a computer simulation study of the square-well model of a simple (atomic) fluid along supercritical isotherms. They make a striking claim, i.e. they have uncovered a "colloidal-like state of a pure one-component fluid" that is "a fourth equilibrium state of matter". Strong stuff!

Authors: Yes.

Referee 2: Close reading of the manuscript, which is obscurely drafted, reveals curious statements/observations that leads one to suspect the authors are critical point /critical phenomena deniers. It will become clear what I mean by this. But a glance at Figure 2, will show that the authors do not accept the view that all fluids that exhibit gas-liquid coexistence will exhibit a unique critical point where the transition becomes continuous along with all the accompanying universality of thermodynamic and long-wavelength structural properties (universal critical exponents) that form a cornerstone of modern condensed matter science.

Authors: Yes, this is indeed the case.

Referee 2: The denial is hidden until the penultimate paragraph of the paper when bizarre mutterings are made regarding the phenomenon of critical opalescence. Are the authors really challenging the very well accepted Ornstein-Zernike (OZ) theory from 1914? Modern versions of this venerable treatment, pioneered by luminaries such as M. E. Fisher, K. G. Wilson, J. Sengers and several top-class experimentalists, have confirmed beyond reasonable doubt that the $\mathrm{OZ}$ analysis of the liquid structure factor at small wavenumbers captures the phenomenon. This is in turn intimately linked to the divergence of the compressibility at the critical point.

Authors: In fact, the Ornstein-Zernike theory is not "well-accepted".

This referee should read the paper by Green, for example, "On the Theory of the Critical Point of a Simple Fluid” Melville S Green, J. Chem. Phys. 33, 1403 (1960)

http://dx.doi.org/10.1063/1.1731419/

"Abstract: The consequences of a new system of integral equations for the theory of the critical point are discussed. Reasons are given for believing that the fundamental assumption of the Ornstein-Zernicke theory about the direct correlation function is incorrect."

The three "luminaries" named here, it seems, may have illuminated mathematical theory of simplified lattice model systems, but these models are largely detached from the mundane reality of liquid-gas experimental 
thermodynamics.

Referee 2: Specific points:

The results presented are for a square-well model with (a narrow) well width $\lambda=0.005 \sigma$. The snapshots in Figure 4 are interesting, but these are not placed in proper context. There is no reference to earlier careful studies of the same model. For example J. Largo et al. J.C.P. 128, 134513 (2008) study, using state-of-art finite size methods, wells of different width and go as low as $\lambda=0.005 \sigma$. They describe the dependence of the (standard thermodynamic) critical point on the well-width and conclude that for $l=0.005 \sigma, T_{c}^{*}=0.2007$ and $\rho_{\mathrm{c}}{ }^{*}=0.542$. Their analysis is based on the machinery associated with of continuous (critical) phase transitions. They have no reason to doubt this does apply in this particular model.

Authors: Our values for the critical parameters agree well with the paper referred to by the referee (Largo, J., Miller, M.A., Sciortino, F, (2008) “The vanishing Limit of the square-well fluid: The adhesive hard-sphere model of a square-well fluid” J. Chem. Phys. 128: 134513 except for their misinterpretation of the "critical density" which is a mean density of liquid and gas phases at the critical temperature.

This is one of many hundreds of papers that we might reference. In such papers, interpretation has been vitiated by the assumption of the existence of a van der Waals hypothetical critical point at the outset. One cannot deal with the inadequacy of this mountain of previous work in a brief Letter to Nature.

Referee 2: In an earlier paper Miller and Frenkel Phys. Rev. Lett. 90, 135702 (2003) (and a later J.C.P. I believe) studied the limit of adhesive (sticky) hard spheres. They determined the (standard) critical point and the line of percolation transitions. The latter meets the coexistence curve at a density considerably below the critical density. In other words there is no connection between percolation and criticality. If the present authors are convinced of the validity of their story why do they not explain what might be wrong with the earlier analyses?

Authors: Miller's simulations are for a model temperature-dependent effective pair potential that is not the same as the present molecular model; indeed is not relevant to the results in our Letter.

Referee 2: Intrigued by the present claims I looked at Refs. [2] [3]. Both papers are by Woodcock. Both make interesting reading and both shed light on the gist of the current submission. J. Phys. Chem. (B) (2012) also deals with square well-models. It contains bizarre statements about a first order transition at the critical point. Moreover, in the penultimate paragraph one finds statements: "the critical point does not exist as such" and “There is no universality of critical phenomena.” Fluid Phase Equilibria (FPE) (2013) deals with the square well model but also with some real fluids. There is a story about percolation lines, and the accompanying densities rho_PB and rho_PA, that is similar to that in the current paper. In Figure 7 \& Figure 9, a "line of critical states" is drawn.

Authors: Yes, the statement "first order transition at the critical point" is "bizarre" but these words are not to be found a reference [2]. For temperatures below $T_{c}$ there is a first-order phase transition but no "critical point" on the Gibbs density surface. For temperatures above $T_{c}$, in the mesophase region, there is a one-phase system of a colloidal like nature.

Referee 2: I know no researcher in the field of liquids, let alone any expert in critical phenomena, who would take this seriously. Indeed most would become apoplectic as these papers demonstrate a basic lack of understanding of the thermodynamics and of the experimental literature. I also looked at another paper in FPE (2013) by Heyes and Woodcock. This is on Lennard-Jones fluids but the thrust is much the same. The authors speak of an alternative description of liquid-gas criticality and argue there is no critical singularity. In the final section, they refer to the Nobel lecture of Wilson where he describes the notions of universality. It is abundantly clear the authors have no understanding of the fundamental concepts and the intellectual framework underpinning the whole subject of critical phenomena. No serious referee would have accepted these papers. In summary this paper contributes nothing to our understanding of fluids near critical points.

Authors: This statement contains no cogent scientific argument. When did referees ever become "apoplectic" we ask, on having to review demonstrably bad science? The medical condition, apoplexy, is more likely a symptom of referees having to face up to home truths about their own shortcomings in science comprehension.

Referee 2: Rather it is the latest in a series by one of the authors who appears to be convinced that the whole subject of critical point behavior has been misunderstood or wrongly interpreted.

Authors: Yes.

Referee 2: Given that some of the best minds in physics have contributed to our current understanding it is a brave, or misguided, person who makes such a call.

Authors: There has never been an “understanding”, only a misunderstanding. Van der Waals' undefined and 
hypothetical "critical point” has always been at variance with Gibbs thermodynamics. Any understanding must be both thermodynamic and at the molecular level. Why, we ask, is it "misguided” to call into question hypotheses that do not address the thermodynamic description of gas-liquid equilibria for temperatures at or above $T_{c}$ with any proper definitions?

Referee 2: Summary: there is no evidence in the current work to suggest that the large community of experimentalists, simulators and theorists studying critical phenomena has got it completely wrong. Recommendation: reject.

Referee 3: The authors assert that there has “...been recent suggestion that at temperatures above the critical there is no 'continuity of liquid and gas'...” However, it should be noted that the recent suggestion has been by the author himself in Ref. [2]. The main difference between this current manuscript and the previously published work is the use of square well fluids with short-range attraction (making cluster counting unambiguous). The authors call these adhesive hard spheres.

Authors: Yes.

Referee 3: The authors are proposing that there is no continuity between liquid and gas at the liquid-gas critical point, but rather that there is a mesophase between liquid and gas at supercritical conditions defined by percolation transitions. Molecular dynamics simulations of hard sphere plus attractive square well are performed and analyzed to support this hypothesis.

Authors: Yes.

Referee 3: First, I find this paper most unconvincing. Nothing in the simulation details gives me confidence that they are sampling equilibrium configurations, something which becomes increasing difficult as the well width in the square well fluid is reduced.

Authors: All the details of the runs and the program DYNAMO are given. Anyone can repeat our simulations and get the same results. One of the authors (LVW) has 45 years experience of the applications of molecular dynamics (MD) simulation to chemical physics research. We know the difference between EMD and NEMD!

Referee 3: Second, it should not be published in Nature, as the authors have already attempted to justify their rather unusual point of view in at least one previous paper (Ref. [2]). If the idea has merit, it will ultimately be debated in the scientific literature, and found to be right or wrong.

Authors: Now this referee resorts to political arguments against publication; degree-of-novelty policy is a matter for the Nature editor. Ironically, anonymous referee 3 here concedes possibility "idea has merit" and needs to be "debated" whilst cynically obstructing its publication.

Referee 3: Third, I cannot see how one can write a manuscript on this subject and not reference the very careful work of Miller and Frenkel (J. Phys.: Condens. Matter 16 S4901 doi:10.1088/0953-8984/16/42/008) on adhesive hard spheres, who point out the difficulties of molecular dynamics for short-ranged attractive systems. In studying the percolation transition, Miller and Frenkel state “As the threshold is crossed, thermodynamic properties and their derivatives change smoothly, and so percolation is not a thermodynamic phase transition.”

Authors: The simulation referred to was for a system of 864 atoms, Miller and Frenkel do not have any information on thermodynamic-limit systems, their statement is quite incorrect generally (see References [7] [8]). Furthermore, the paper by Miller and Frenkel is irrelevant as it relates to the Baxter model, which does not correspond to any molecular Hamiltonian (the "effective” pair potential is T-dependent); their paper tells us nothing.

Referee 3: Without having studied the problem in detail myself, I am far more inclined to accept Daan Frenkel's conclusion than the contrarian one of these authors. At the very least, Frenkel should be a reviewer of this work.

Authors: We specifically requested his exclusion from any peer-review process of this Letter owing to his record of unprofessional conduct with manuscripts submitted in confidence. Also, he has previously recommended this new science of liquid-gas criticality should not be published without valid scientific reasons. 
Scientific Research Publishing (SCIRP) is one of the largest Open Access journal publishers. It is currently publishing more than 200 open access, online, peer-reviewed journals covering a wide range of academic disciplines. SCIRP serves the worldwide academic communities and contributes to the progress and application of science with its publication.

Other selected journals from SCIRP are listed as below. Submit your manuscript to us via either submit@scirp.org or Online Submission Portal.
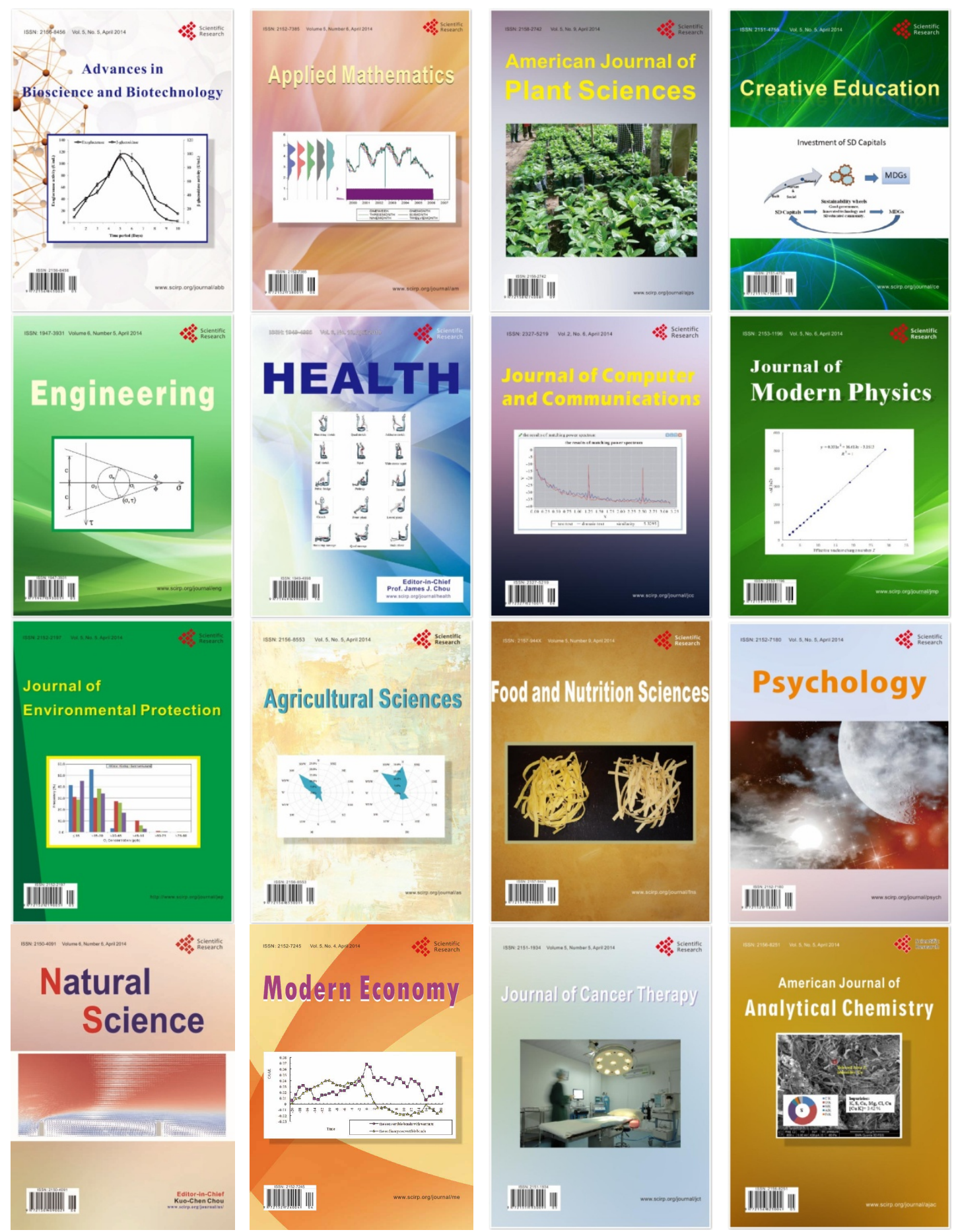